\title{
Room-Temperature Switching of Spin-State in Single-Molecule Junctions
}

\author{
Jing Li \\ Xiamen University \\ QingQing Wu \\ Lancaster University \\ Wei Xu \\ Xiamen University \\ Hai-Chuan Wang \\ Xiamen University \\ Hewei Zhang \\ Xiamen University \\ Yongxiang Tang \\ Songjun Hou
}

Lancaster University

Yaorong Chen

Xiamen University

Colin Lambert

Lancaster University https://orcid.org/0000-0003-2332-9610

Wenjing Hong ( $\nabla$ whong@xmu.edu.cn )

Xiamen University https://orcid.org/0000-0003-4080-6175

\section{Article}

Keywords: molecular spintronics, molecular devices, spin-state, molecular spin switches, roomtemperature switching

Posted Date: September 28th, 2020

DOl: https://doi.org/10.21203/rs.3.rs-78276/v1

License: (9) (1) This work is licensed under a Creative Commons Attribution 4.0 International License. Read Full License 


\title{
Room-Temperature Switching of Spin-State in Single-Molecule Junctions
}

Jing Li, ${ }^{1} \uparrow$ QingQing Wu, ${ }^{2} \uparrow$ Wei Xu,${ }^{1} \uparrow$ Hai-Chuan Wang, ${ }^{1}$ Hewei Zhang,,${ }^{1}$ Yongxiang Tang, ${ }^{1}$ Songjun Hou, ${ }^{2}$ Yaorong Chen, ${ }^{1}$ Colin J. Lambert, ${ }^{2}$ and Wenjing Hong $* 1$

${ }^{1}$ State Key Laboratory of Physical Chemistry of Solid Surfaces, College of Chemistry and Chemical Engineering, Xiamen University, Xiamen, China

${ }^{2}$ Department of Physics, Lancaster University, Lancaster, UK

$\uparrow$ These authors contributed equally to this work.

\begin{abstract}
The emerging of molecular spintronics offers a unique chance for the design of molecular devices with different spin-state, and the control of spin-state becomes essential for molecular spin switches. However, the intrinsic spin switching from low-spin to high-spin state is a temperature-dependent process with a small energy barrier that low temperature is required to maintain the low-spin state, and thus the room-temperature operation of single-molecule devices have not yet been achieved. Here, we investigated the single-molecule charge transport through a diamagnetic square planar nickel(II) porphyrin using the scanning tunneling microscope break-junction (STM-BJ) technique. The reversible single-molecule conductance switches are demonstrated by utilizing a coordinationinduced spin-state switching to manipulate the spin state between $S=0$ and $S=1$ at room temperature. Furthermore, the different coordinated complexes could be distinguished from the conductance traces, which cannot be realized by the ensemble investigations such as NMR and UVvis spectrums. The combined DFT calculations revealed that the conductance changes come from the different spin-states of the molecules varying the number of coordination ligands, suggesting coordination-induced spin-state switching provides a new way towards room-temperature molecular spintronics.
\end{abstract}

\section{Introduction}

Molecular spintronics is an emerging field that aims to exceed the limits of conventional electronics by utilizing the spin of the electrons, which has many advantages such as non-volatility, high storage density, low energy consumption, and high response speed. ${ }^{1-5}$ As one of the prototype devices, molecular spin switches, especially intrinsic spin switches, which can be switched between at least two stable spin-states by external stimuli such as temperature, ${ }^{6}$ pressure,${ }^{7}$ photons,${ }^{6,8}$ electric fields, ${ }^{9}$ 
etc., have been considered as the essential step towards the development of molecular spintronics. ${ }^{10-}$ ${ }^{13}$ Recently, several types of single-molecule prototype devices have been demonstrated, such as voltage-triggered, ${ }^{14}$ stretching-induced ${ }^{15,16}$ or electron injection induced ${ }^{17-19}$ spin switches. However, due to the difference in spin multiplicity and vibrational levels, the phase transition of intrinsic spin switch from low-spin (LS) to high-spin (HS) is an entropy-increasing process $(\Delta S>0)$, suggesting that the transition is temperature-dependent, and the LS state is more stable at low temperature. ${ }^{20,21}$ Thus, the low-temperature vacuum conditions are required to obatin a stable, low-spin initial state on an electrode surface, and the increasing of their operating temperature to room temperature remains challenging.

To realize room-temperature manipulation of spin-state in single-molecule devices, the interfacial interaction between molecule and electrode is another major issue. ${ }^{22-24}$ After adsorption on electrode surfaces, the intrinsic spin switch functionality may be lost or changed (such as, a lower phase transition temperature), which brings difficulty for the molecular design. To address this issue, coordination-induced spin-state switching (CISSS), ${ }^{25}$ which is insensitive to interfacial interaction, provides a promising strategy for the control of spin-state via the coordinated at room temperature. The spin-states of some $3 \mathrm{~d}$ metal ions ${ }^{25-27}$ are sensitive to the coordination of the geometric structure, and thus the squar planar Ni-center diamagnetic low-spin state will be switched-on $(S=0 \rightarrow S=1)$ by controlling axial ligand coordination to a square pyramidal or octahedral configuration. ${ }^{18,25,28-30}$ On the other hand, previous studies have also demonstated that the charge transport through the metal porphyrin could determine the different elements and even charge states of the metal center, ${ }^{31-}$ ${ }^{33}$ suggesting the control of nickel porphyrin between different spin-state may offer a promising way towards single-molecule spin switches at room temperature via CISSS strategy.

In this work, we designed a square planar nickel(II) porphyrin derivative, 5,15-bis-(4methylthiophenyl)-10,20-bis-(2,3,4,5,6-pentafluorophenyl)-Ni(II) porphyrin (NiTPPF, Figure 1A), and investigated its spin-induced single-molecule conductance switching using STM-BJ technique at room temperature. Without axial ligands, NiTPPF exhibits a diamagnetic low-spin state $(S=0)$ with an empty $d_{x^{2}-y^{2}}$ and a fully occupied $d_{z^{2}}$ orbital of the central nickel ion. One electron is transferred from the $d_{z^{2}}$ orbital to the $d_{x^{2}-y^{2}}$ orbital leading to a paramagnetic high-spin species $(S=1)$ upon axial coordination with at least one 3, 5-Lutidine molecule (Figure 1A). Compared with charge transport through the $\mathrm{Zn}(\mathrm{II})$-analogue, a giant conductance switch was achieved by manipulating the spin-states, which is comparable with most of the single-molecule magnetoresistance systems requiring low temperature and ultra-high vacuum. Furthermore, we found that the five- and sixcoordination NiTPPF complexes can be identified by their single-molecule conductance, which cannot be done with the ensemble investigations such as NMR and UV-vis spectra. These spin 
manipulations are further confirmed by density functional theory calculation.

A

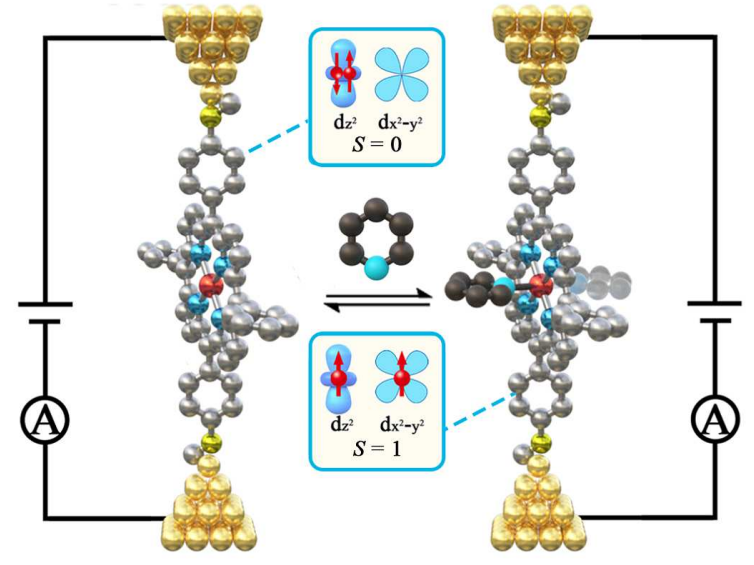

C

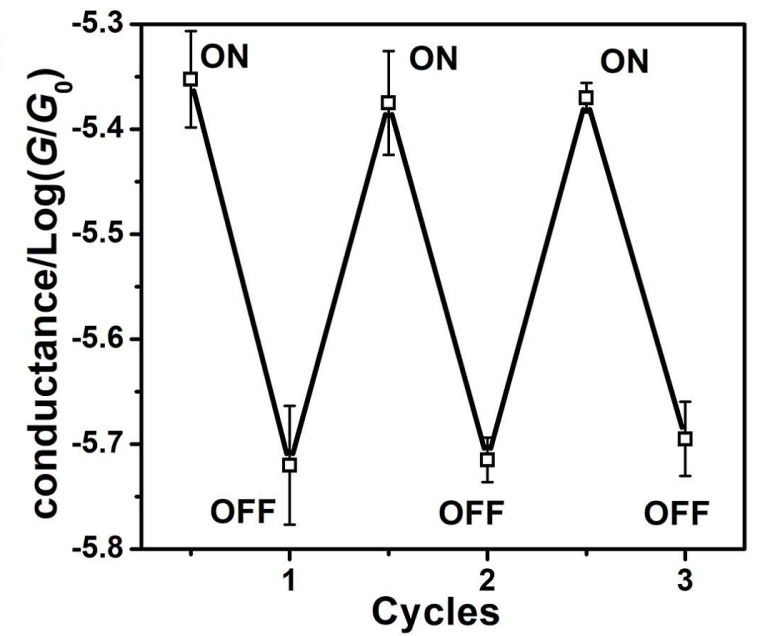

B

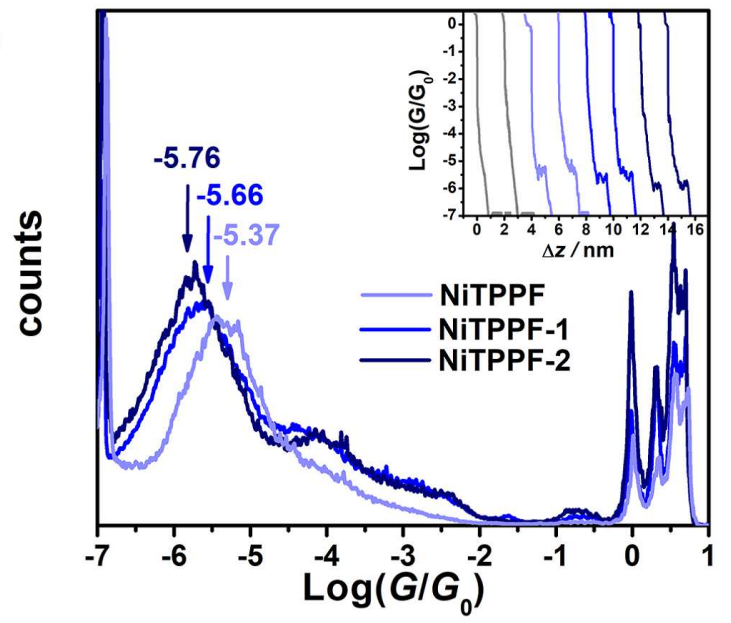

D

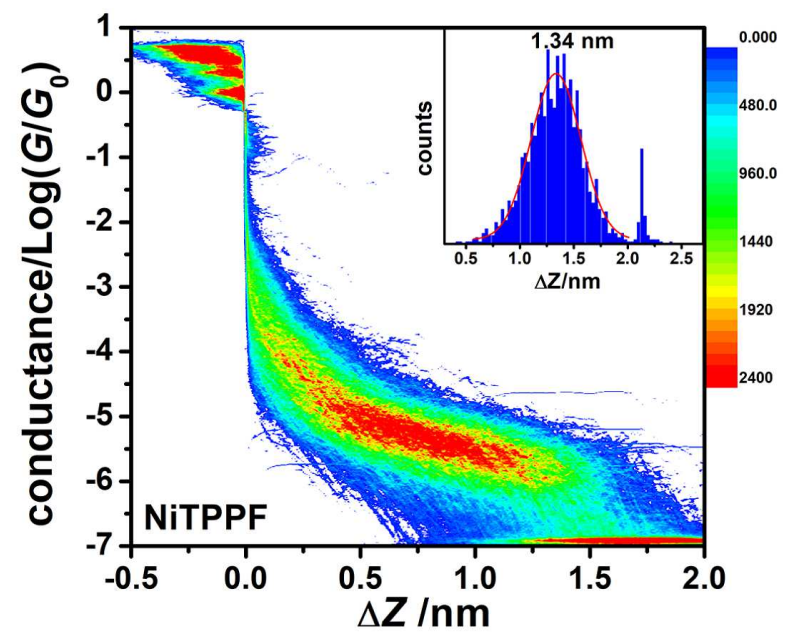

Figure 1 | The single molecule conductance properties of NiTPPF and axial coordinated derivate. A, Contact geometries for the NiTPPF series of molecules. The reversible coordination reaction between NiTPPF and 3, 5-Lutidine in solution, and the related spin switch $(S=0 \leftrightarrow S=1)$. The $\mathrm{H}$ and $\mathrm{F}$ atoms in NiTPPF, and methyl group in 3,5-Lutidine are omitted. B, Logarithmically binned conductance histograms of NiTPPF (3869 traces), NiTPPF-1 (2788 trace), and NiTPPF-2 (2377 traces), respectively. Inset: Individual conductance traces measured in solutions of NiTPPF, NiTPPF-1 and NiTPPF-2 at 0.1V, respectively. C, Reversible on-off conductance switches of the NiTPPF system with adding 3, 5-Lutindine and $\mathrm{CF}_{3} \mathrm{COOH}$. D, $2 \mathrm{D}$ conductance histograms generated from 3869 conductance traces measured at $0.1 \mathrm{~V}$ for NiTPPF. Inset: the plateau length of NiTPPF

\section{Results and discussion}

NMR and UV-Vis spectrum in ensemble solution. Pentafluorophenyl nickel(II) porphyrin 
derivatives are often used to study CISSS in references. ${ }^{25,28-30}$ Here, we designed a new porphyrin NiTPPF, which has two -SMe anchor groups at opposite ends of the molecule, to enable charge transport studies. The NMR spectroscopy and UV-vis spectra of NiTPPF and its mixture with the electron-rich auxiliary ligand 3, 5-Lutidine were collected at room temperature to monitor the axial coordination reaction process (Supplementary Figure 1). One index from low spin diamagnetic Ni(II) transitioning to a high-spin state $(S=1)$ is whether the chemical shift of the pyrrole proton to a low field with larger ppm value, giving rise to a five or six coordinated complex. ${ }^{25,28-30}$ From their NMR spectroscopy, the chemical shifts of the pyrrole protons in the pure NiTPPF are located in $\sim 8.8$ and $8.5 \mathrm{ppm}$ (Supplementary Figure 2A), which shifted to lower fields after titrating with 3, 5-Lutidine, indicating the formation of paramagnetic five- and six-coordinated complexes (Supplementary Figure 2B). However, only an average chemical shift of the three components (NiTPPF, five- and six-coordinated NiTPPF) can be observed in the spectroscopy, because of the fast equilibrium of complexation and decomplexation. ${ }^{25,28,29}$ The UV-vis titration spectra show that the band of pure NiTPPF at $415.8 \mathrm{~nm}$ decreases and a new band arises at $433.1 \mathrm{~nm}$ upon the addition of 3, 5-Lutidine (Supplementary Figure 3A), which is consistent with reference. ${ }^{29}$ Although we cannot distinguish the five- and six-coordinated complexes from the NMR and UV-vis titration methods, both spectra demonstrate the CISSS in NiTPPF and 3, 5-Lutidine solutions.

Single-molecule conductance measurements. To characterize the charge transport through NiTPPF and its axial coordinated derivative molecules, the conductance of single-molecule junctions was measured using the STM-BJ technique. ${ }^{34-37}$ We measured three solutions which are $0.1 \mathrm{mM}$ pure NiTPPF solution, $0.1 \mathrm{mM}$ pure NiTPPF solution with 1.1 equivalent 3, 5-Lutidine (NiTPPF-1), and with 2.2 equivalent 3, 5-Lutidine (NiTPPF-2), respectively. For each solution, we repeatedly form and break gold-gold atom contacts. During the gold tip retraction, a plateau around $\mathrm{G}_{0}\left(2 \mathrm{e}^{2} / \mathrm{h}, 77.6\right.$ $\mu \mathrm{S}$ ) representing a gold-gold atom contact can be observed first, followed by some other plateaus below $\mathrm{G}_{0}$. These additional plateaus indicate the formation of $\mathrm{Au}$-molecule-Au junctions.

For each experiment, we collected thousands of conductance traces $(3869,2788$, and 2377 traces for NiTPPF, NiTPPF-1, and NiTPPF-2, respectively) to analyze the 1D conductance histograms (Figure 1B) and 2D conductance-displacement histograms (Figure 1D, and Supplementary Figures 5, 7). All complexes showed a prominent low conductance peak and a less prominent high conductance peak. ${ }^{38}$ The measured plateaus lengths can be fitted with a Gaussian function. The plateau length of high conductance junctions is ca. $0.42 \mathrm{~nm}$, which is nearly half-length of the whole molecule (ca. $0.92 \mathrm{~nm}$ ), after adding a $0.5 \mathrm{~nm}$ gold-gold snap back distance to the stretching distance (Supplementary Figure 5), which suggests that this plateau occurs when one of the gold tips connects with the porphyrin ring. ${ }^{38}$ The plateau length corresponding to the low-conductance junctions is ca. $1.4 \mathrm{~nm}$. Hence, the corrected junction length is ca. $1.9 \mathrm{~nm}$, which is in line with the sulfur-sulfur 
distance of NiTPPF. Therefore, the low conductance peak for each sample contains the core information for studying the CISSS-induced charge transport features. The most probable conductance value for each sample is obtained by fitting a Gaussian function to the conductance peak. As shown in Figure 1B, the low conductance of the isolated NiTPPF is $10^{-5.37} \mathrm{G}_{0}(0.331 \mathrm{nS})$. The conductance shifts to $10^{-5.66} \mathrm{G}_{0}(0.170 \mathrm{nS})$ for NiTPPF-1, and further shifts to a lower value $10^{-}$ ${ }^{5.76} \mathrm{G}_{0}(0.135 \mathrm{nS})$ for NiTPPF-2. We can define a parameter $R$, analogous to magnetoresistance effect, ${ }^{39}$ to evaluate the efficiency of resistance switching rate in the CISSS behavior, defined using the following formula:

$$
R(\%)=\frac{R_{\mathrm{HS}}-R_{\mathrm{LS}}}{R_{\mathrm{LS}}}=\frac{S_{\mathrm{LS}}-S_{\mathrm{HS}}}{S_{\mathrm{HS}}} \times 100(1)
$$

where $R_{\mathrm{HS}}, R_{\mathrm{LS}}, S_{\mathrm{HS}}$, and $S_{\mathrm{LS}}$ represent the resistance or conductance of high-spin and low-spin states, respectively. In this system, the value of $R$ reached $145.8 \%$ with increasing the 3, 5-Lutidine to 2.2 equivalents. The magnitude of $R$ in the present experiments is comparable with most of the singlemolecule magnetoresistance systems reported in the literature. ${ }^{39,40}$

To evaluate the effect of spin manipulation on charge transport, we further measured the singlemolecule conductance of ZnTPPF as a control experiment, which does not change the spin-state after adding an extra axial ligand (Supplementary Figure 8). The UV-vis spectra show that the band of pure ZnTPPF at $425 \mathrm{~nm}$ shifts to $431 \mathrm{~nm}$ upon the addition of 3, 5-Lutidine (Supplementary Figure 3B), indicating the five- or six-coordinated complex was formed. ${ }^{41}$ The $1 \mathrm{D}$ conductance histograms and 2D conductance-displacement cloud maps were similar to NiTPPF. The conductance of the pure $\mathbf{Z n T P P F}$ is about $10^{-5.26} \mathrm{G}_{0}(0.426 \mathrm{nS})$, which is slightly larger than NiTPPF, and consistent with previously reported systems. ${ }^{31}$ After adding 3, 5-Lutidine, the conductance of coordinated ZnTPPF decreased to $10^{-5.31} \mathrm{G}_{0}(0.380 \mathrm{~ns})$ for ZnTPPF-1, and $10^{-5.35} \mathrm{G}_{0}(0.347 \mathrm{~ns})$ for ZnTPPF-2. The slight decrease of about $22.8 \%$ can be attributed to the small energy change in electron energy levels after axial-coordination. From another perspective, the rate is only one-fifth of the NiTPPF system (Supplementary Figure 9), which reveals that the spin switching plays a crucial role in charge transport.

Benefiting from the stability of NiTPPF in the solution of $\mathrm{CF}_{3} \mathrm{COOH}$ at room temperature, ${ }^{[11 \mathrm{c}]}$ the five- or six-coordinated NiTPPF can decomplexate to pure NiTPPF by adding excess $\mathrm{CF}_{3} \mathrm{COOH}$ to completely protonate the 3,5 -Lutidine. As a result, the high spin state $(S=1)$ can be switched back to low spin state $(S=0)$. Through adding $\mathrm{F}_{3} \mathrm{COOH}$ or 3, 5- Lutidine in the samples, we observed the conductance switching in a reversible way (Figure 1C). 
A
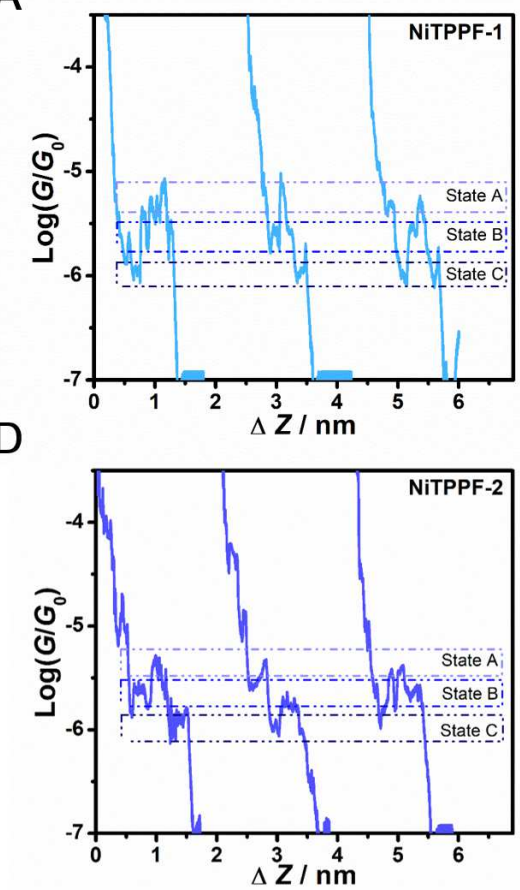

B

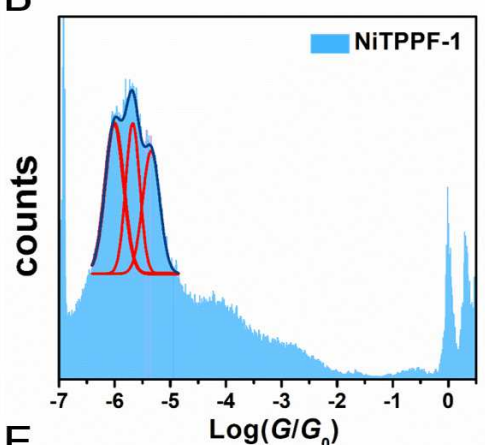

E

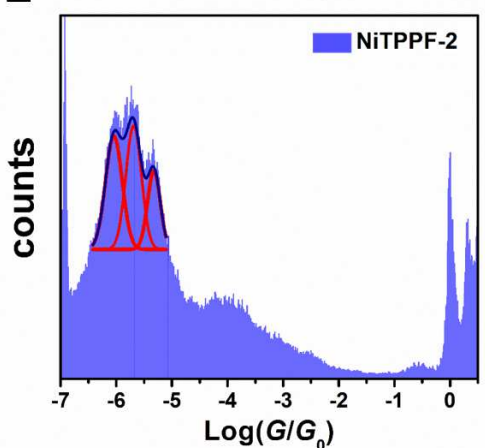

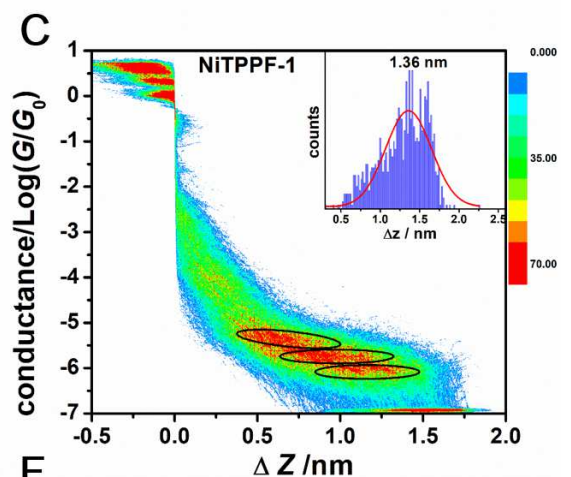

$\mathrm{F}$

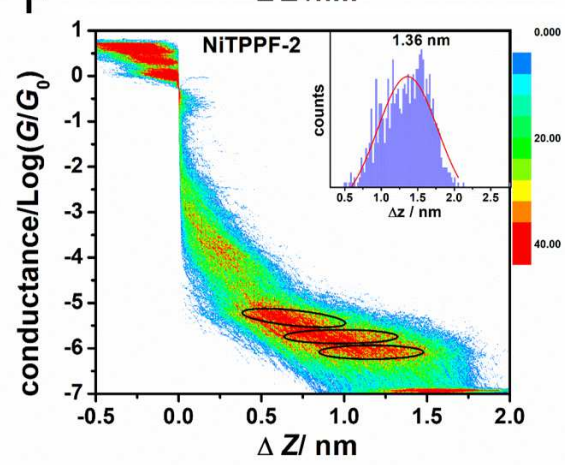

Figure 2 | The analysis results based on single conductance traces. A, D, The observed three different states in single conductance traces for NiTPPF-1 and NiTPPF-2, respectively. B, E, Logarithmically binned conductance histograms of NiTPPF-1 (20.6\%, 1011 out of 4898 traces) and NiTPPF-2 $(20.3 \%, 1152$ out of 5658 traces) filtered by a plateau with a duration of six milliseconds, respectively. C, F, 2D conductance histograms generated from the filtered traces for NiTPPF-1 and NiTPPF-2, respectively. Inset: the plateau length of related molecules.

The conductance of different structures. In the mixed solution of NiTPPF and 3, 5-Lutidine, NMR spectroscopy (Supplementary Figure 2B) shows that the complexation and decomplexation reaction is continuous (Figure 1A, and Supplementary Figure 1) and forms a chemical equilibrium. As a result, when the STM-BJ experiment is carried out, each one of the components in the solution can form a single-molecule junction between the gold-gold nanogap. To further explore the charge transport of individual components, we focus on the conductance behaviors in the single trace. Interestingly, three conductance plateaus can be observed in most single traces, which is in line with an ensemble chemical reaction mechanism. An algorithm (see in Supplementary information section V) was programmed to select the traces containing all three conductance.

We assume the conductance of the three components are $10^{-5.3 \pm 0.1} \mathrm{G}_{0}(\mathrm{~A}), 10^{-5.71 \pm 0.1} \mathrm{G}_{0}(\mathrm{~B})$, and $10^{-}$ ${ }^{6.1 \pm 0.1} \mathrm{G}_{0}(\mathrm{C})$, according the conductance traces in Figure 2A, 2D and Supplementary Figure 10. Then the target traces can be obtained by defining the plateau length. If a plateau with a duration of 3 milliseconds (60 single shots) is regarded as the conductance of one component, about 59.4\% (2907 in 4898) and 69.1\% (3914 in 5658) of all traces contain three conductance for NiTPPF-1 and 
NiTPPF-2, respectively (Supplementary Table 1). When the time is extended to $6 \mathrm{~ms}$ (120 single shots), $20.6 \%$ (1011 in 4898) and 20.3\% (1152 in 5658) of all traces remain, respectively. Three peaks can be observed in the 1D conductance histogram shown in Figure 2B and 2E, and those peaks were gradually covered as the screening conditions are relaxed (Supplementary Figure 11). In the three peaks, the highest conductance peak is centered around $10^{-5.32} \mathrm{G}_{0}(0.371 \mathrm{~ns})$ for NiTPPF-1, and $10^{-5.34} \mathrm{G}_{0}(0.355 \mathrm{~ns})$ for NiTPPF-2, which is well in agreement with the conductance of pure NiTPPF. Thus, the two lower conductance peaks corresponding to the five- or six-coordinated complexes (Figure 1A and supplementary Figure 1). The centers of the 1D conductance histogram are around $10^{-5.68} \mathrm{G}_{0}(0.162 \mathrm{nS})$ and $10^{-6.01} \mathrm{G}_{0}(0.075 \mathrm{nS})$ for NiTPP-1, and $10^{-5.68} \mathrm{G}_{0}(0.162 \mathrm{nS})$ and $10^{-6.04} \mathrm{G}_{0}(0.071 \mathrm{nS})$ for NiTPP-2. Based on the above analysis (Supplementary Table 2), the resistance of the high-spin state can be enhanced up to $400 \%$ compared to the diamagnetic state. From the single trace analysis, we can capture the dynamic processes of the axial-coordinated reaction, and distinguish the five- or six- coordinated complexes through STM-BJ technique, which cannot be distinguished from the ensemble investigations such as NMR and UV-vis spectrums.
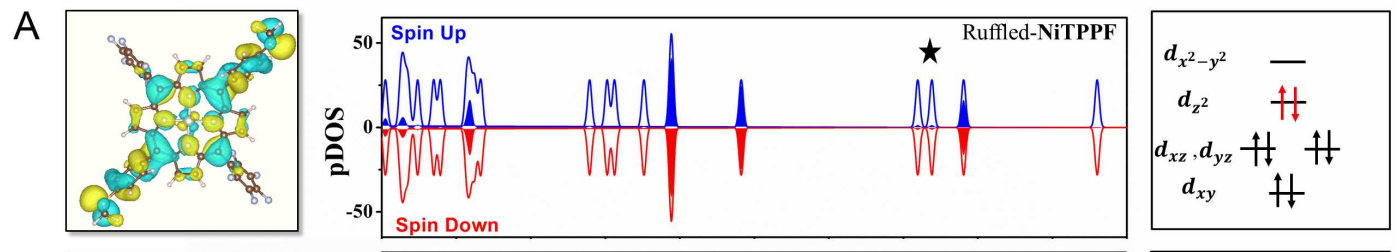

B
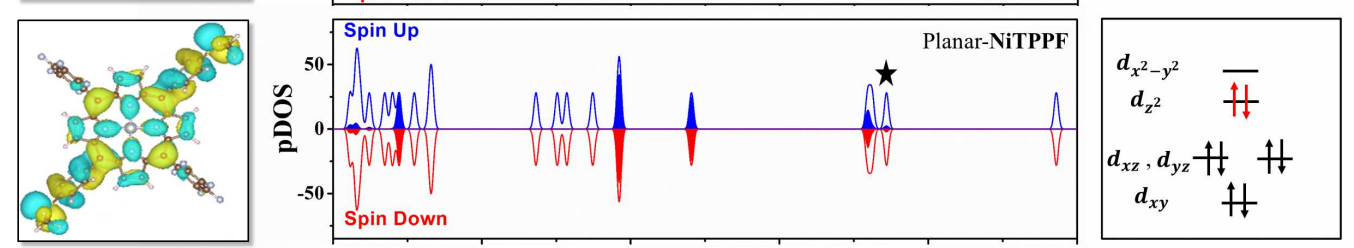

C
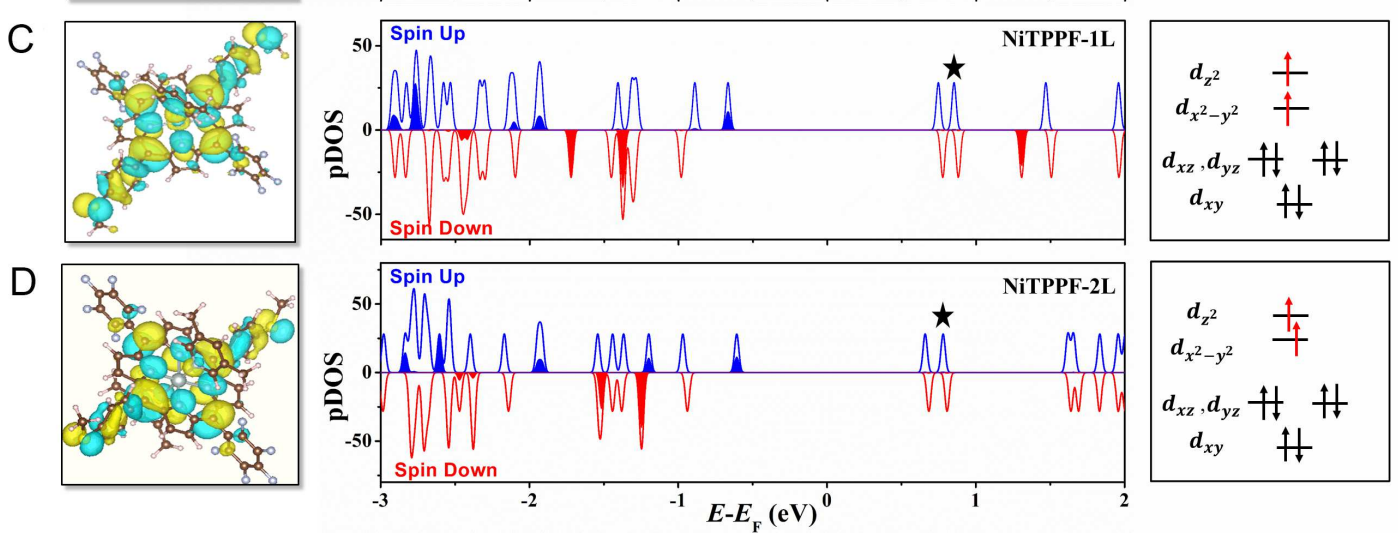

Figure 3 | The DFT calculated spin-resolved projected density of states of gas-phase NiTPPF series of molecules. Blue and red colors correspond to the spin-up and spin-down contributions, respectively. The filled curve corresponds to the metal orbital of $\mathrm{Ni}$ ion, and their occupations are shown in the right side. The isosurface plots of the star marked orbitals are given on the left sides. 
Theoretical calculation. To provide insight into the experimental results, theoretical simulations and analysis based on density functional theory, a tight-binding model, and quantum transport theory are carried out for the four molecules (ruffled-NiTPPF, planar-NiTPPF, five-coordinated NiTPPF-1L, six-coordinated NiTPPF-2L). ${ }^{42,43}$ The DFT calculated spin-resolved projected density of states of the four complexes are summarized in Figure 3. Generally, the pure NiTPPF molecules exhibit a ruffled geometry in crystal lattices, while a planar geometry is possible in the gas-phase and the solvent. Hence, both of the geometries were examined (Figure 3A and B). The calculated results show that ruffled and planar geometries are both in the low-spin ground states $(S=0)$, and the former is more energetically favorable than the latter by $20 \mathrm{meV}$ lower total energy. Four $d$ orbitals $\left(d_{x y}\right.$, $d_{y z}, d_{x z}, d_{z^{2}}$ ) of Ni atom are fully occupied, whereas the $d_{x^{2}-y^{2}}$ orbital moves to a higher energy and becomes the LUMO (the lowest unoccupied molecular orbital) for the planar molecule. Considering the axial coordination by one and two 3,5-Lutidine, the ruffled porphyrin tends to be planar, with a high-spin ground state $(S=1)$.

The PDOS plotted in Figure 3C-D shows the similar alignments and occupations of the NiTPPF in the presence of one and two 3, 5-Lutidine ligands, for which $d_{x^{2}-y^{2}}$ of one spin component is occupied and $d_{z^{2}}$ of the other spin is empty and moves to higher energy. The wave functions which are mainly located on the porphyrin and barely on the anchor parts (Supplementary Figure 12,13) would have a limited role in transmission functions. The orbitals closest to the Fermi energy are considered to form the pronounced resonances in transmission functions which have weights delocalized on the whole molecule including the -SMe anchors (It is worth noting that the Fermi energy is not physically meaningful for gas-phase molecules). To facilitate observation, the relevant orbitals are marked by stars in the density of states spectrum, and their isosurface plots are given on the left and right sides (Supplementary Figure 14). The eight important orbitals marked by stars have quite similar shapes and are all distributed on the whole molecules, indicating that the $3 \mathrm{~d}$ orbitals of $\mathrm{Ni}$ ions play a perturbing role in transmission functions. 
A

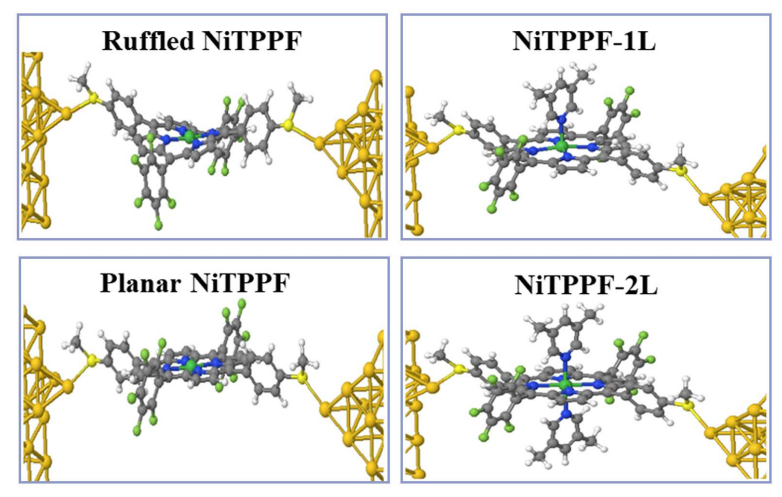

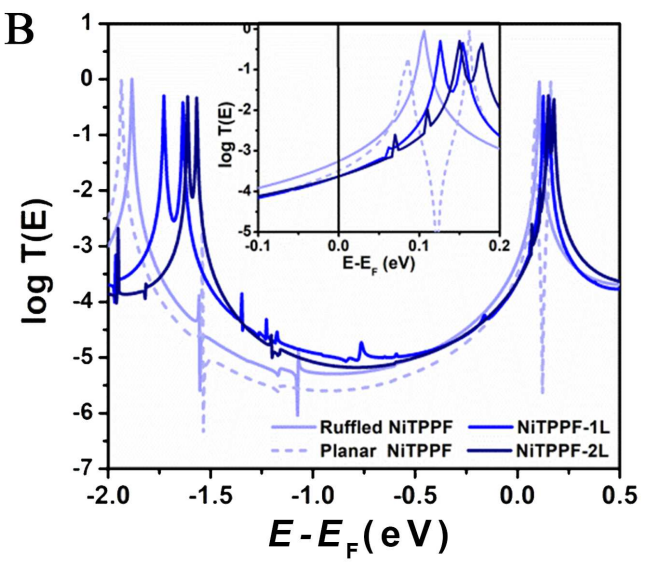

Figure 4 | Geometry models and transmission functions in simulations. A, Configurations of gold/NiTPPF-series/gold junctions. B, The transmission functions of the junctions. The curves of NiTPPF-1L and NiTPPF-2L depict the average transmission functions over the two spin channels where the two molecules have a high-spin ground state

For each of these structures, the configurations and corresponding transmission functions of the gold/molecule/gold junctions for the four molecules were presented and analyzed (Figure 4). It should be noted that the curves of NiTPPF-1L and NiTPPF-2L shown in Figure 4 are the average transmission spectra over spin up and down components (Supplementary Figure 15), so the two neighboring peaks between $-2 \mathrm{eV}$ and $-1.5 \mathrm{eV}$ belong to the two spin channels. The curves of ruffled and planar-NiTPPF molecules possess wider energy gaps between the peaks around $-2 \mathrm{eV}$ and 0.2 eV compared to those of NiTPPF-1L and NiTPPF-2L due to the quantum effect of different molecular sizes. The very narrow resonances at $-1.5 \mathrm{eV}$ and $-1.1 \mathrm{eV}$ are associated with the three $3 \mathrm{~d}$ orbitals $\left(d_{x y}, d_{y z}, d_{x z}\right)$ of Ni for the ruffled and planar-NiTPPF molecules shown in Figure 3A-B. The tiny one at $-1.4 \mathrm{eV}$ in the curve of NiTPPF-1L involves the $d_{x^{2}-y^{2}}$ orbital of the spin-up component. Other kinks between $-1.3 \mathrm{eV}$ and $-0.5 \mathrm{eV}$ are due to instabilities in the calculations. Those very narrow and tiny peaks can be observed both in low-spin and high-spin states which originate from the localized orbitals on porphyrin rings and $\mathrm{Ni}$ atoms and rare weights on the -SMe anchor groups (Supplementary Figure 12,13). Some orbitals are even completely silent, such as, the $d_{z^{2}}$ orbital of the planar-NiTPPF molecule, $d_{x^{2}-y^{2}}$ orbital of spin-up for the NiTPPF-2L molecule.

In the inset of Figure 4B, these pronounced resonances are associated with the orbitals marked by stars in Figure 3. The Fano-resonance at $0.1 \mathrm{eV}$ of the planar-NiTPPF arises from the orbital containing large components of $d_{x^{2}-y^{2}}$, which is localized on the porphyrin ring and the Ni atom and can be rationalized using a tight-binding model (Supplementary Figure 16). The Fermi energy is 
close to the LUMO in all four molecules, suggesting that LUMOs are the dominated charge transport channels. The transmission coefficients of planar and ruffled NiTPPF near the Fermi energy level are larger than those of five- or six-coordinated NiTPPF complexes. Those features reveal the decreasing trend in conductance between the low-spin and high-spin states. We also calculated the $\mathrm{Zn}(\mathrm{II})$-analogue system (Supplementary Figure 17, 18), and demonstrated that the small energy change in electron energy levels after axial coordination can slightly suppress charge transport. However, this effect is weaker than that of the Ni system with spin manipulation, which is in good agreement with the experiments.

\section{Conclusions}

In summary, we demonstrate the reversible switching of spin-state in single-molecule junctions at room temperature using STM-BJ technique, and we have achieved a conductance difference of up to $400 \%$ for different spin-state using coordination-induced spin-state switching. We further proved that the single-molecule conductance is an effective method to study rapid time-scale reactions beyond the ensemble measurements. On the basis of this study, we suggest two possible ways to improve the device. First, from the theoretical calculation, the contribution of $3 \mathrm{~d}$ orbitals of nickel ions to electron transmission is suppressed by the highly conjugated porphyrin ring. Hence, taking advantage of chemical tunability to eliminate the conjugation of the ligand and increase the contribution of the metal ion in the conductive channel will hopefully result in a device with a higher conductance difference. Second, the auxiliary ligand can be functionalized so that it can respond to more stimuli to develop a multifunctional device. For example, photon-driven coordination-induced spin-state switching (LD-CISSS) single-molecule electric device can be explored by introducing a photonactive ligand such as azopyridine derivatives. ${ }^{25,28}$ Therefore, we confidently anticipate that the CISSS concept provides a promising route towards room-temperature single-molecule spintronics, which is of potential for the future development of spin-based molecular memory devices.

\section{Methods}

All reagents were purchased from TCI and used without further purification. The NMR spectrums were recorded on a Bruker $400 \mathrm{M}$ instruments and Tetramethylsilane (TMS) was used as an internal standard.

Synthesis of 5-(Pentafluorophenyl)-dipyrromethane (DPMF)

DPMF was synthesized followed by a reported literature procedure. ${ }^{38,44}$

A solution of Pentafluorobenaldehyde (3.92 g, $20 \mathrm{mmol})$ and an excess amount of pyrrole $(50 \mathrm{~mL})$ was degassed with $\mathrm{N}_{2}$ at room temperature. To this, was added trifluoroacetic acid (TFA) $(0.17 \mathrm{~mL}, 2$ 
mmol) and the reaction mixture was stirred for $30 \mathrm{~min}$ at room temperature. After the reaction, the mixture was treated with $0.1 \mathrm{M} \mathrm{NaOH}$ Solution and then extracted with ethyl acetate for three times. The combined solution was concentrated and purified by column chromatography over silica gel using DCM: Hex = 1:2 as eluent to give product $(1.51 \mathrm{~g}, 24 \%)$ as white solid. ${ }^{1} \mathrm{HNMR}(400 \mathrm{MHz}$, $\left.\mathrm{CDCl}_{3}\right): \delta=5.90(\mathrm{~s}, 1 \mathrm{H}), 6.02(\mathrm{~m}, 2 \mathrm{H}), 6.15-6.17(\mathrm{~m}, 2 \mathrm{H}), 6.73-6.74(\mathrm{~m}, 2 \mathrm{H}), 8.15(\mathrm{~s}, \mathrm{br}, 2 \mathrm{H}) \mathrm{ppm}$.

Synthesis of 5,15-bis(4-(methylthio)phenyl)-10,20-bis(2,3,4,5,6-pentafluorophenyl)-21H,23H porphyrin (TPPF)

Under nitrogen atmosphere, 4-(methylthio)benzldehyde $(0.624 \mathrm{~g}, 2 \mathrm{mmol})$ and 5(pentafluorophenyl)-dipyrromethane were added to dichloromethane $(20 \mathrm{~mL})$ via syringe. Trifluoro acetic acid (0.34 mg, $2 \mathrm{mmol})$ was dissolved in dichloromethane $(5 \mathrm{~mL})$ and added to the solution. The reaction mixture was stirred at $45^{\circ} \mathrm{C}$ for $1.5 \mathrm{~h}$. Then 2,3 -dicyano-5,6-dichlorobenzoquinone was added and stirred for additional $1 \mathrm{~h}$. After the reaction, triethylamine $(2 \mathrm{~mL})$ was added and the solvent was removed by evaporation. The mixture was further purified with column chromatography over silica gel using $\mathrm{DCM} / \mathrm{Hex}=1: 3$ as eluent to get the crude product. The pure crystal product (234 mg, 26\%) was obtained by recrystallized from DCM/MeOH. ${ }^{1} \mathrm{HNMR}\left(400 \mathrm{MHz}, \mathrm{CDCl}_{3}\right): \delta=$ $2.83(\mathrm{~s}, 2 \mathrm{H}), 2.77(\mathrm{~s}, 6 \mathrm{H}), 7.65(\mathrm{~d}, \mathrm{~J}=8.12 \mathrm{~Hz}, 4 \mathrm{H}), 8.13(\mathrm{~d}, \mathrm{~J}=8.12 \mathrm{~Hz}, 4 \mathrm{H}), 8.79$ (d, J = 4.8 Hz, 4H), $8.97(\mathrm{~d}, \mathrm{~J}=4.8 \mathrm{~Hz}, 4 \mathrm{H}) \mathrm{ppm}$.

Synthesis of 5,15-bis(4-(methylthio)phenyl)-10,20-bis(2,3,4,5,6-pentafluorophenyl)Ni(II) porphyrin (NiTPPF)

5,15-bis(4-(methylthio)phenyl)-10,20-bis(2,3,4,5,6-pentafluorophenyl)-21H,23H porphyrin (TPPF) (71 mg, $0.08 \mathrm{mmol})$ and $\mathrm{Ni}(\mathrm{OAc})_{2} \cdot 4 \mathrm{H}_{2} \mathrm{O}(60 \mathrm{mg}, 0.24 \mathrm{mmol})$ was dissolved in $\mathrm{DMF}(10 \mathrm{~mL})$. Then, the mixture was refluxed for $6 \mathrm{~h}$. After the reaction, the mixture was concentrated and washed with water to remove the excess of $\mathrm{Ni}(\mathrm{OAc})_{2} \cdot 2 \mathrm{H}_{2} \mathrm{O}$. The crude product was further purified with column chromatography over silica gel using $\mathrm{DCM} / \mathrm{Hex}=1: 5$ as eluent to obtain product as red solid $(27 \mathrm{mg}$, 36\%). ${ }^{1} \mathrm{HNMR}\left(400 \mathrm{MHz}, \mathrm{CDCl}_{3}\right): \delta=2.73(\mathrm{~s}, 6 \mathrm{H}), 7.59(\mathrm{~d}, \mathrm{~J}=8.24 \mathrm{~Hz}, 4 \mathrm{H}), 7.95$ (d, J = 8.16 Hz, 4H), $8.68(\mathrm{~d}, \mathrm{~J}=4.8 \mathrm{~Hz}, 4 \mathrm{H}), 8.88(\mathrm{~d}, \mathrm{~J}=5.08 \mathrm{~Hz}, 4 \mathrm{H}) \mathrm{ppm}$.

Synthesis of 5,15-bis(4-(methylthio)phenyl)-10,20-bis(2,3,4,5,6-pentafluorophenyl)Zn(II) porphyrin (ZnTPPF)

5,15-bis(4-(methylthio)phenyl)-10,20-bis(2,3,4,5,6-pentafluorophenyl)-21H,23H porphyrin (TPPF) (44 mg, $0.05 \mathrm{mmol})$ and $\mathrm{Zn}(\mathrm{OAc})_{2} \cdot 2 \mathrm{H}_{2} \mathrm{O}(39 \mathrm{mg}, 0.175 \mathrm{mmol})$ was dissolved in $\mathrm{CHCl}_{3} / \mathrm{MeOH}=2: 1(9 \mathrm{~mL})$. Then, the mixture was refluxed for $3 \mathrm{~h}$. After the reaction, the mixture was concentrated and filtrated through a short silica gel column with ethyl acetate as eluent. The 
solvent was evaporated and the resulting purple solid was washed with $\mathrm{MeOH}$ to obtain pure product (45 mg, 98\%). 1 HNMR (400 MHz, Toluene-D8): $\delta=2.29$ (s, 6H), 7.52 (d, J = 8.12 Hz, 4H), 7.95 (d, $\mathrm{J}=8.12 \mathrm{~Hz}, 4 \mathrm{H}), 8.68(\mathrm{~d}, \mathrm{~J}=4.76 \mathrm{~Hz}, 4 \mathrm{H}), 8.88(\mathrm{~d}, \mathrm{~J}=4.6 \mathrm{~Hz}, 4 \mathrm{H}) \mathrm{ppm}$.

NMR titration. Each ${ }^{1} \mathrm{HNMR}$ tube was filled with $0.2 \mathrm{~mL}$ of a NiTPPF solution $(10.0 \mathrm{mmol} / \mathrm{L})$ in toluene-d8. Then different amounts of a solution of ligand in toluene-d8 (10 vol\%) were added and the tubes were filled up to $0.3 \mathrm{~mL}$ with toluene- $\mathrm{d} 8$ and sealed. We measured five different ligand concentration solutions $(0.2 \mathrm{mmol} / \mathrm{L}, 0.4 \mathrm{mmol} / \mathrm{L}, 0.6 \mathrm{mmol} / \mathrm{L}, 0.8 \mathrm{mmol} / \mathrm{L}$ and $1.0 \mathrm{mmol} / \mathrm{L})$.

Conductance measurements: Conductance measurements were performed using the home-built STM through the break-junction technique to form gold-molecule-gold junctions as previous reported at room temperature. A detailed STMBJ experiments, a detailed explanation of the conductance of different structures can be found in the supplementary information file.

Computational Methods. Geometrical optimizations were performed using the standard Kohn-Sham self-consistent density functional code SIESTA, ${ }^{43}$ with GGA functional and a double- $\zeta$ polarized atomic-orbital basis sets. The cut-off energy was $200 \mathrm{Ry}$, and the force tolerance was $0.01 \mathrm{eV} / \AA$ for the gas phase molecules and $0.02 \mathrm{eV} / \AA$ for the junctions. To compute their electrical conductance, the molecule was placed between two gold electrodes. For each structure, the transmission coefficient $T(E)$ describing the propagation of electrons of energy $E$ from lead 1 to lead 2 was calculated using the Gollum quantum transport code, ${ }^{42}$ which utilizes the DFT mean-field Hamiltonian and overlap matrices from SIESTA and computes $T(\mathrm{E})$ via the following formula

$$
T(E)=\operatorname{Tr}\left[\Gamma_{1}(E) g(E) \Gamma_{2}(E) g^{\dagger}(E)\right]
$$

where $g(E)=\left(g_{0}^{-1}-\Sigma_{1}-\Sigma_{2}\right)^{-1}$ is the retarded Green's function in the presence of the electrodes and $\Gamma_{j}(E)=i\left(\Sigma_{j}(E)-{\Sigma_{j}}^{\dagger}(E)\right) / 2$, is the anti-Hermitian part of the self-energies $\Sigma_{j}$, which encode the electronic structure of the semi-infinite electrodes and molecule and the electrode-molecule interface. $\Gamma_{j}$ determines the broadening of transmission resonances due to the contact between the molecule and electrode $j . g_{0}$ is the Green's function of isolated molecule. Finally, the transmission coefficient between lead 1 and lead 2, $T_{12}(E)$, was obtained for electrons of energy $E$ travelling separately from lead 1 to the other leads.

\section{References}

1 Wolf, S. A. et al. Spintronics: A Spin-Based Electronics Vision for the Future. Science 294, 1488-1495, doi:10.1126/science.1065389 (2001).

2 Xiang, D., Wang, X., Jia, C., Lee, T. \& Guo, X. Molecular-Scale Electronics: From Concept to Function. Chem. Rev. 116, 4318-4440, doi:10.1021/acs.chemrev.5b00680 (2016). 
Sanvito, S. Molecular spintronics. Chem. Soc. Rev. 40, 3336-3355, doi:10.1039/C1CS15047B (2011).

Aradhya, S. V. \& Venkataraman, L. Single-molecule junctions beyond electronic transport. Nat. Nanotechol. 8, 399-410, doi:10.1038/nnano.2013.91 (2013).

Choi, S. H., Kim, B. \& Frisbie, C. D. Electrical resistance of long conjugated molecular wires. Science 320, 14821486, doi:10.1126/science.1156538 (2008).

Cheng, X. et al. Thermal and light induced spin crossover behavior of a dinuclear Fe(ii) compound. Dalton Transactions 44, 11282-11285, doi:10.1039/C5DT01755F (2015).

Pinkowicz, D. et al. Enforcing Multifunctionality: A Pressure-Induced Spin-Crossover Photomagnet. J. Am. Chem. Soc. 137, 8795-8802, doi:10.1021/jacs.5b04303 (2015).

Wang, H.-Y. et al. Photo- and Electronically Switchable Spin-Crossover Iron(II) Metal-Organic Frameworks Based on a Tetrathiafulvalene Ligand. Angew. Chem. Int. Ed. 56, 5465-5470, doi:10.1002/anie.201611824 (2017).

Mahfoud, T. et al. Electric-Field-Induced Charge-Transfer Phase Transition: A Promising Approach Toward Electrically Switchable Devices. J. Am. Chem. Soc. 131, 15049-15054, doi:10.1021/ja9055855 (2009).

10 Ruben, M. \& Kumar, K. S. Sublimable Spin Crossover Complexes: From Spin-State Switching to Molecular Devices. Angew. Chem. Int. Ed. doi:10.1002/anie.201911256 (2019).

1 Bairagi, K. et al. Molecular-scale dynamics of light-induced spin cross-over in a two-dimensional layer. Nat. Commun. 7, 12212, doi:10.1038/ncomms12212 (2016).

2 Miyamachi, T. et al. Robust spin crossover and memristance across a single molecule. Nat. Commun. 3, 938, doi:10.1038/ncomms1940 (2012).

3 Han, Y. et al. Electric-field-driven dual-functional molecular switches in tunnel junctions. Nat. Mater. 19, 843848, doi:10.1038/s41563-020-0697-5 (2020).

4 Harzmann, G. D., Frisenda, R., van der Zant, H. S. \& Mayor, M. Single-Molecule Spin Switch Based on VoltageTriggered Distortion of the Coordination Sphere. Angew. Chem. Int. Ed. 54, 13425-13430, doi:10.1002/anie.201505447 (2015).

5 Frisenda, R. et al. Stretching-Induced Conductance Increase in a Spin-Crossover Molecule. Nano Lett. 16, 47334737, doi:10.1021/acs.nanolett.5b04899 (2016).

Parks, J. J. et al. Mechanical Control of Spin States in Spin-1 Molecules and the Underscreened Kondo Effect. Science 328, 1370-1373, doi:10.1126/science.1186874 (2010).

1 Brandl, T. et al. Iron in a Cage: Fixation of a Fe(II)tpy2 Complex by Fourfold Interlinking. Angew. Chem. Int. Ed. doi:10.1002/anie.202006340 (2020).

Kobke, A. et al. Reversible coordination-induced spin-state switching in complexes on metal surfaces. Nat. Nanotechol. 15, 18-21, doi:10.1038/s41565-019-0594-8 (2020).

Gopakumar, T. G. et al. Electron-induced spin crossover of single molecules in a bilayer on gold. Angew. Chem. Int. Ed. 51, 6262-6266, doi:10.1002/anie.201201203 (2012).

Hauser, A., Jeftić, J., Romstedt, H., Hinek, R. \& Spiering, H. Cooperative phenomena and light-induced bistability in iron(II) spin-crossover compounds. Coord. Chem. Rev. 190-192, 471-491, doi:10.1016/S0010-8545(99)001113 (1999).

1 Bernien, M. et al. Highly Efficient Thermal and Light-Induced Spin-State Switching of an Fe(II) C

2 Cinchetti, M., Dediu, V. A. \& Hueso, L. E. Activating the molecular spinterface. Nat. Mater. 16, 507-515, doi:10.1038/nmat4902 (2017).

3 Carlotti, M. et al. Two-Terminal Molecular Memory through Reversible Switching of Quantum Interference Features in Tunneling Junctions. Angew. Chem. Int. Ed. 57, 15681-15685, doi:10.1002/anie.201807879 (2018). Aragonès, A. C. et al. Metal-Controlled Magnetoresistance at Room Temperature in Single-Molecule Devices. J. 
Am. Chem. Soc. 139, 5768-5778, doi:10.1021/jacs.6b11166 (2017).

Venkataramani, S. et al. Magnetic Bistability of Molecules in Homogeneous Solution at Room Temperature. Science 331, 445-448, doi:10.1126/science.1201180 (2011).

Wackerlin, C. et al. Controlling spins in adsorbed molecules by a chemical switch. Nat. Commun. 1, 61, doi:10.1038/ncomms1057 (2010).

7 Shankar, S. et al. Light-controlled switching of the spin state of iron(III). Nat. Commun. 9, 4750, doi:10.1038/s41467-018-07023-1 (2018).

Thies, S. et al. Light-Induced Spin Change by Photodissociable External Ligands: A New Principle for Magnetic Switching of Molecules. J. Am. Chem. Soc. 133, 16243-16250, doi:10.1021/ja206812f (2011).

9 Thies, S. et al. Coordination-induced spin crossover (CISCO) through axial bonding of substituted pyridines to nickel-porphyrins: sigma-donor versus pi-acceptor effects. Eur. J. Chem. 16, 10074-10083, doi:10.1002/chem.201000603 (2010).

Gutzeit, F. et al. Structure and Properties of a Five-Coordinate Nickel(II) Porphyrin. Inorg. Chem. 58, 1254212546, doi:10.1021/acs.inorgchem.9b00348 (2019).

1 Liu, Z. F. et al. Control of single-molecule junction conductance of porphyrins via a transition-metal center. Nano Lett. 14, 5365-5370, doi:10.1021/nl5025062 (2014).

Sedghi, G. et al. Long-range electron tunnelling in oligo-porphyrin molecular wires. Nat. Nanotechol. 6, 517-523, doi:10.1038/nnano.2011.111 (2011).

Leary, E. et al. Bias-Driven Conductance Increase with Length in Porphyrin Tapes. J. Am. Chem. Soc. 140, 1287712883, doi:10.1021/jacs.8b06338 (2018).

4 Xu, B. \& Tao, N. J. Measurement of single-molecule resistance by repeated formation of molecular junctions. science 301, 1221-1223 (2003).

5 Venkataraman, L., Klare, J. E., Nuckolls, C., Hybertsen, M. S. \& Steigerwald, M. L. Dependence of singlemolecule junction conductance on molecular conformation. Nature 442, 904-907, doi:10.1038/nature05037 (2006).

6 Cui, X. D. et al. Reproducible Measurement of Single-Molecule Conductivity. Science 294, 571-574, doi:10.1126/science.1064354 (2001).

7 Aragonès, A. C. et al. Electrostatic catalysis of a Diels-Alder reaction. Nature 531, 88-91, doi:10.1038/nature16989 (2016).

8 Leary, E. et al. Detecting Mechanochemical Atropisomerization within an STM Break Junction. J. Am. Chem. Soc. 140, 710-718, doi:10.1021/jacs.7b10542 (2018).

9 Schmaus, S. et al. Giant magnetoresistance through a single molecule. Nat. Nanotechol. 6, 185-189, doi:10.1038/nnano.2011.11 (2011).

Yang, K. et al. Tunable giant magnetoresistance in a single-molecule junction. Nat. Commun. 10, 3599, doi:10.1038/s41467-019-11587-x (2019).

1 Kojima, T. et al. Impact of Distortion of Porphyrins on Axial Coordination in (Porphyrinato)zinc(II) Complexes with Aminopyridines as Axial Ligands. Eur. J. Inorg. Chem. 2009, 727-734, doi:10.1002/ejic.200801040 (2009).

2 Ferrer, J. et al. GOLLUM: a next-generation simulation tool for electron, thermal and spin transport. New Journal of Physics 16, 093029, doi:10.1088/1367-2630/16/9/093029 (2014).

3 Soler, J. M. et al. The SIESTA method forab initioorder-Nmaterials simulation. J. Phys.: Condens. Matter 14, 2745-2779, doi:10.1088/0953-8984/14/11/302 (2002).

4 Fujii, S. et al. Highly-conducting molecular circuits based on antiaromaticity. Nat. Commun. 8, 15984, doi:10.1038/ncomms15984 (2017). 
Acknowledgements. This work is supported by the National Natural Science Foun-dation of China (21722305, 21673195, 21973079 and 21703188), and the National Key R\&D Program of China (2017YFA0204902). This work is also supported by FET Open project 767187-QuIET, the EU project BAC-TO-FUEL, and the UK EPSRC grants EP/N017188/1 and EP/M014452/1 in Lancaster.

Author Contributions. W. H. and J. L. designed the project; W. H. coordinated the project; J. L. and H.-C. W. performed the STM-BJ experiments; W. X. synthesized the molecules; J. L., H. Z, Y. T., and Y. C. analyzed the data; Q. W., S. H., and C. J. L. carried out the theoretical calculation; W. H., J. L., and C. J. L. drafted the manuscript; All authors have given approval to the manuscript.

\section{Competing Interests}

The authors declare no competing interests.

\section{Additional Information}

Supplementary Information accompanies this paper at https://doi.org/10.1038/xxxxxx.

Author Information. Reprints and permissions information is available at www.nature.com/reprints. The authors declare no competing financial interests. Readers are welcome to comment on the online version of the paper. Publisher's note: Springer Nature remains neutral with regard to jurisdictional claims in published maps and institutional affiliations. Correspondence and requests for materials should be addressed to Wenjing Hong. (whong@xmu.edu.cn). 
A

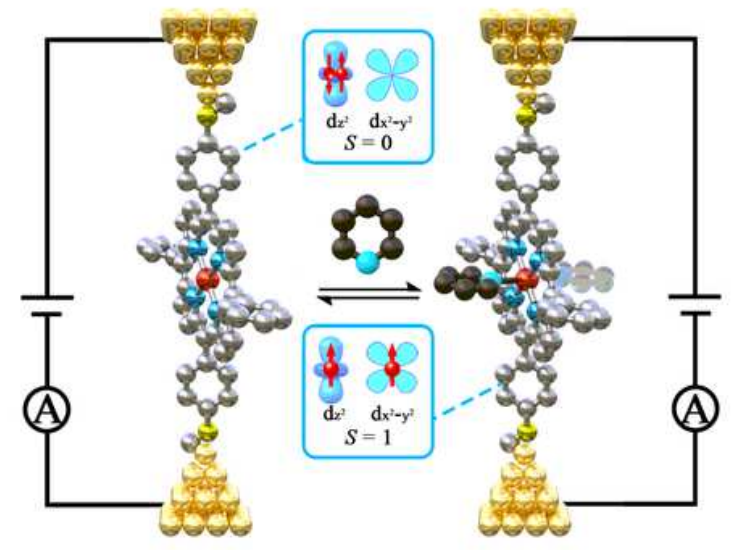

C

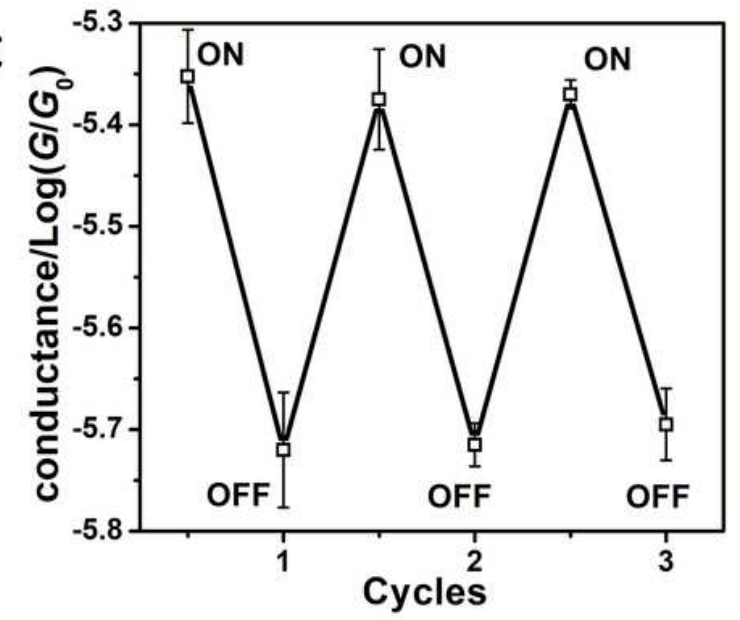

B

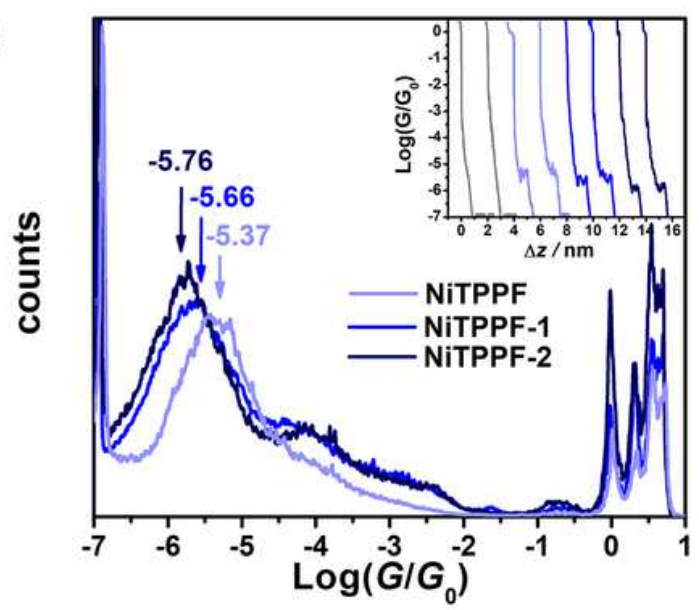

D

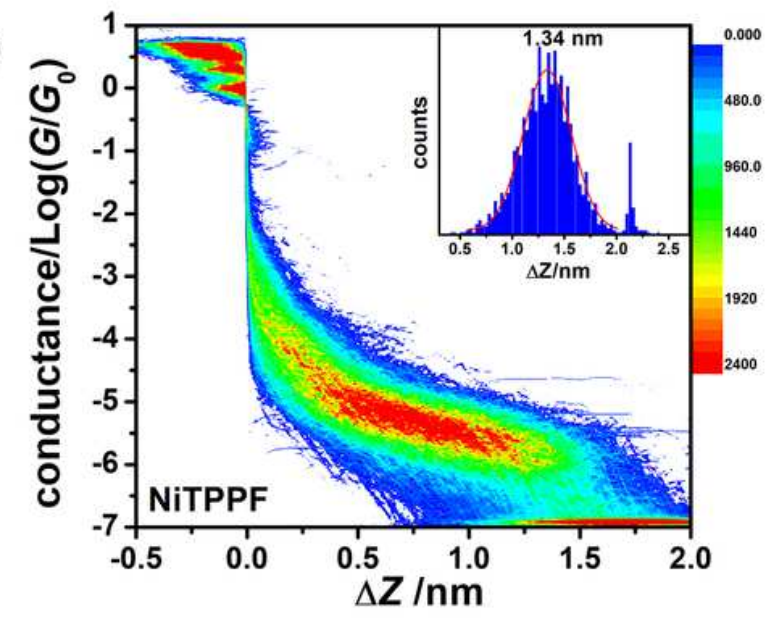

Figure 1

The single molecule conductance properties of NiTPPF and axial coordinated derivate. A, Contact geometries for the NiTPPF series of molecules. The reversible coordination reaction between NiTPPF and 3 , 5-Lutidine in solution, and the related spin switch $(S=0 \otimes S=1)$. The $\mathrm{H}$ and $\mathrm{F}$ atoms in NiTPPF, and methyl group in 3,5-Lutidine are omitted. B, Logarithmically binned conductance histograms of NiTPPF (3869 traces), NiTPPF-1 (2788 trace), and NiTPPF-2 (2377 traces), respectively. Inset: Individual conductance traces measured in solutions of NiTPPF, NiTPPF- 1 and NiTPPF-2 at $0.1 \mathrm{~V}$, respectively. C, Reversible on-off conductance switches of the NiTPPF system with adding 3, 5-Lutindine and CF3COOH. $D, 2 D$ conductance histograms generated from 3869 conductance traces measured at $0.1 \mathrm{~V}$ for NiTPPF. Inset: the plateau length of NiTPPF 
A

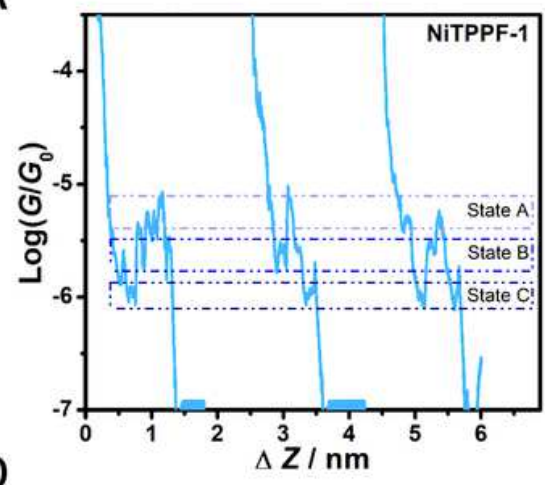

D

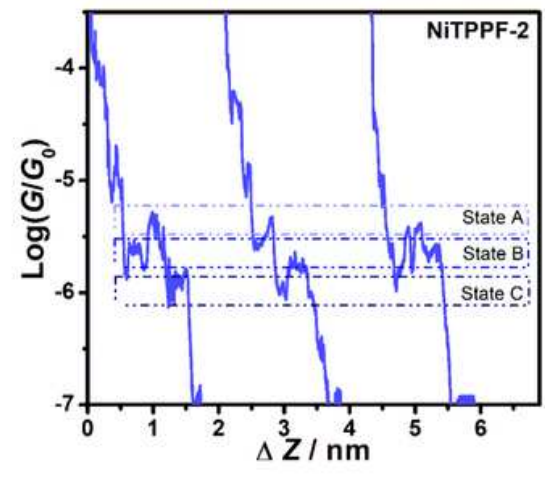

B
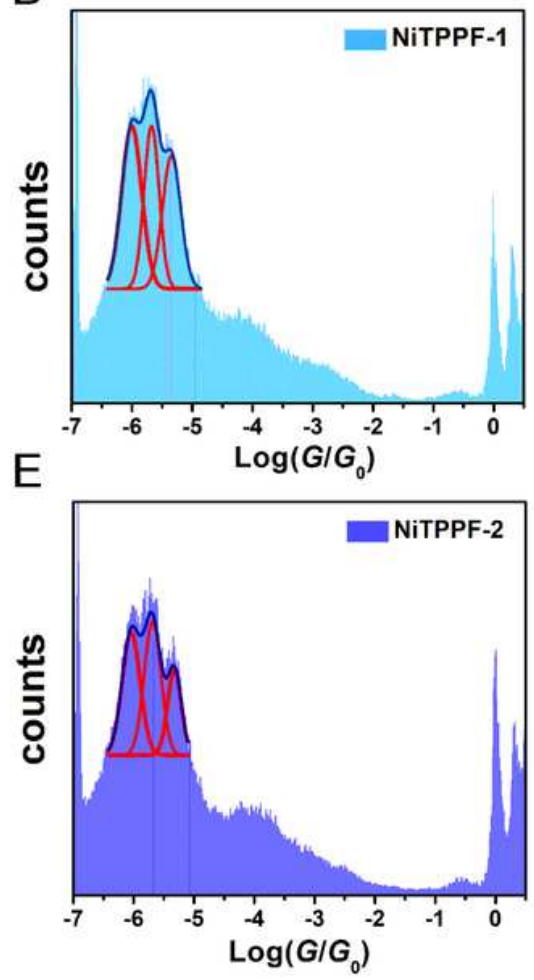

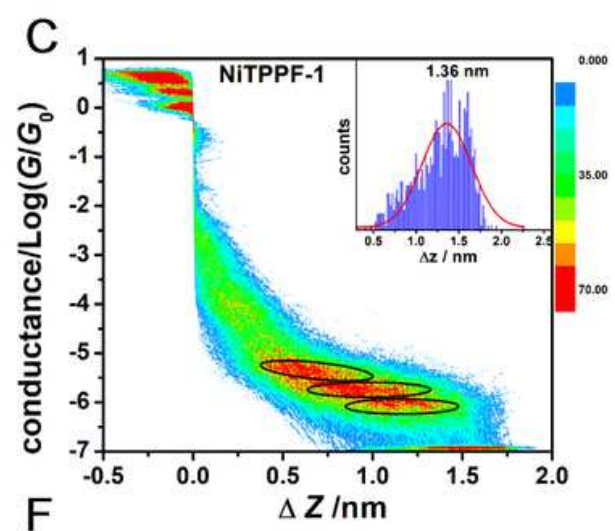

$\mathrm{F}$

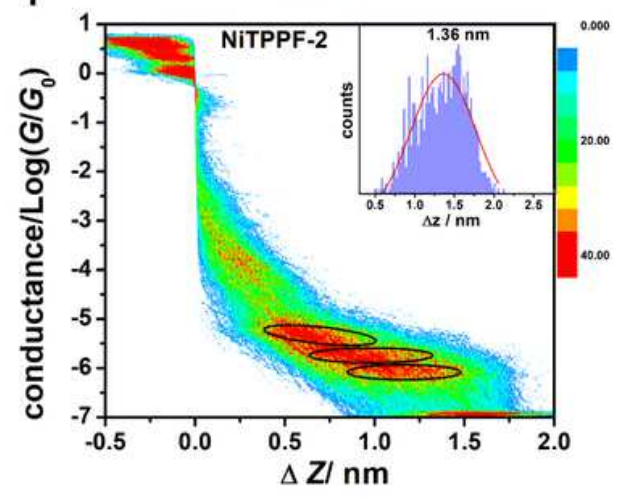

Figure 2

The analysis results based on single conductance traces. A, D, The observed three different states in single conductance traces for NiTPPF-1 and NiTPPF-2, respectively. B, E, Logarithmically binned conductance histograms of NiTPPF- 1 (20.6\%, 1011 out of 4898 traces) and NiTPPF-2 (20.3\%, 1152 out of 5658 traces) filtered by a plateau with a duration of six milliseconds, respectively. C, F, 2D conductance histograms generated from the filtered traces for NiTPPF-1 and NiTPPF-2, respectively. Inset: the plateau length of related molecules. 

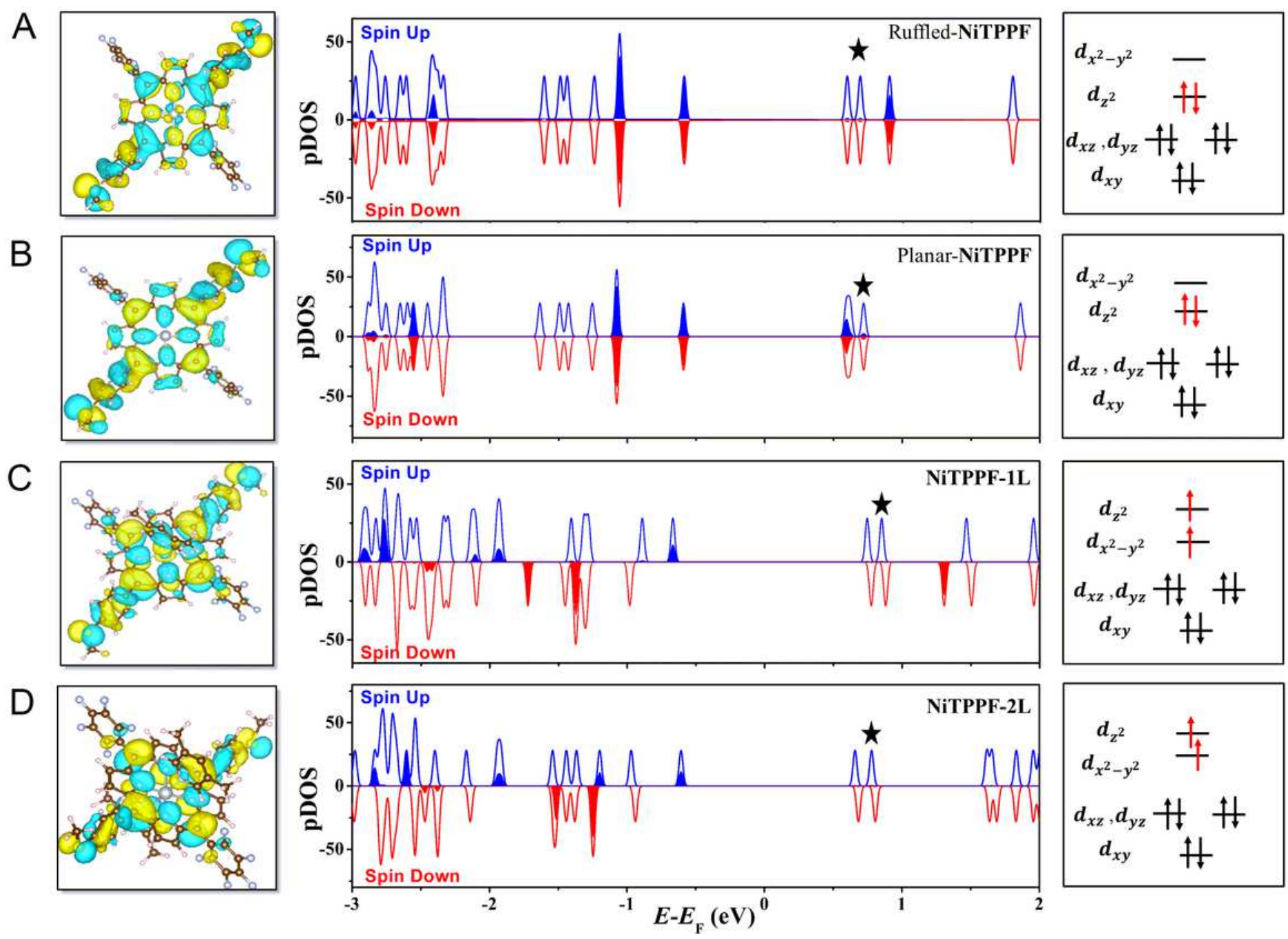

Figure 3

The DFT calculated spin-resolved projected density of states of gas-phase NiTPPF series of molecules. Blue and red colors correspond to the spin-up and spin-down contributions, respectively. The filled curve corresponds to the metal orbital of $\mathrm{Ni}$ ion, and their occupations are shown in the right side. The isosurface plots of the star marked orbitals are given on the left sides. 
A
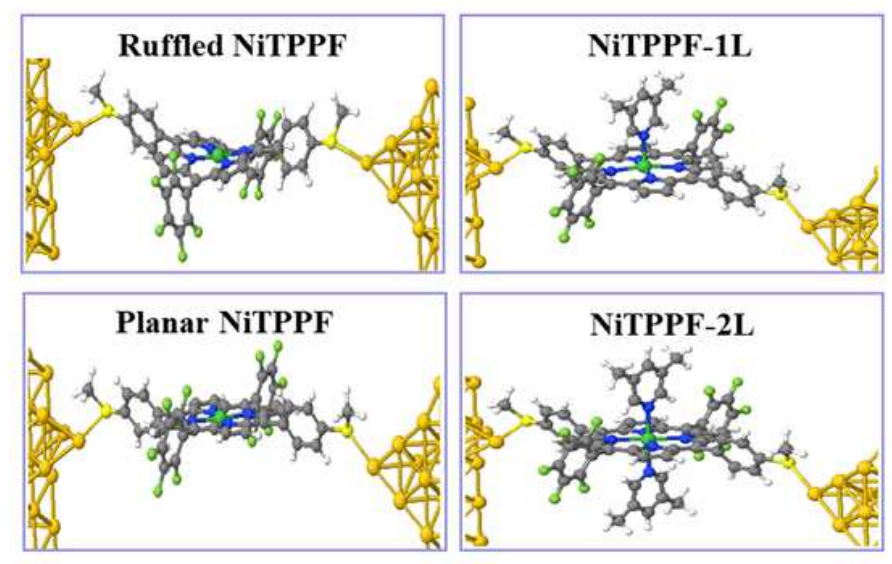

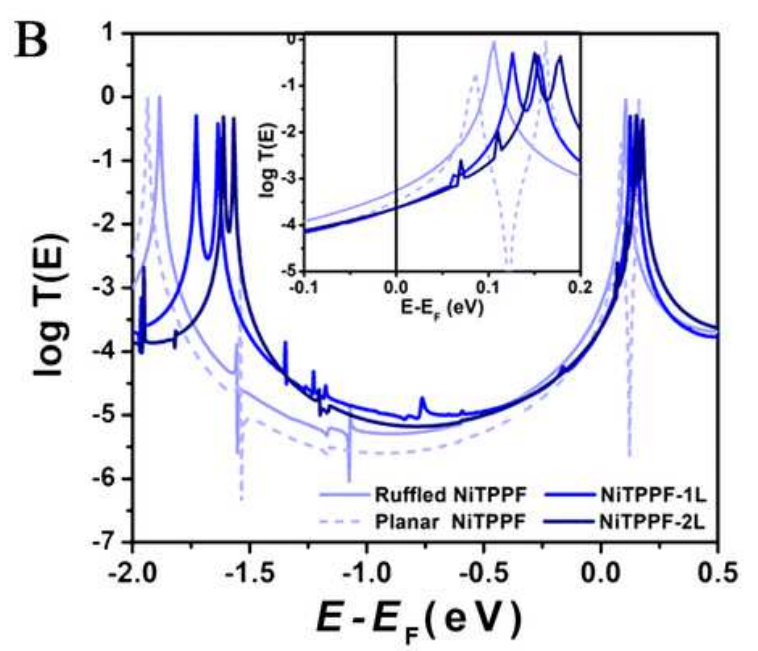

\section{Figure 4}

Geometry models and transmission functions in simulations. A, Configurations of gold/NiTPPFseries/gold junctions. B, The transmission functions of the junctions. The curves of NiTPPF-1L and NiTPPF-2L depict the average transmission functions over the two spin channels where the two molecules have a high-spin ground state

\section{Supplementary Files}

This is a list of supplementary files associated with this preprint. Click to download.

- SInc.docx 